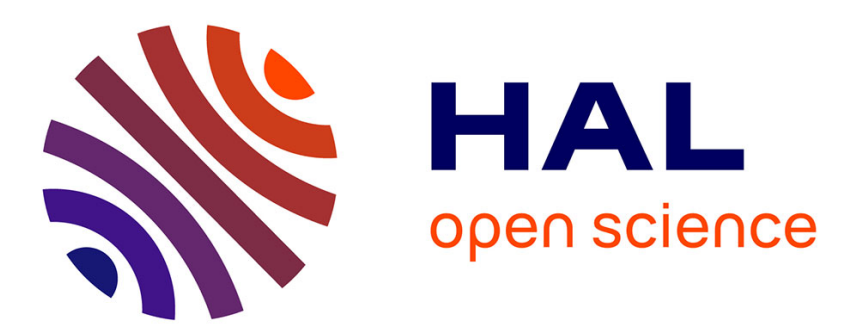

\title{
Esquisse de typologie des noms d'affect à partir de leurs propriétés combinatoires
}

\author{
Agnès Tutin, Iva Novakova, Francis Grossmann, Cristelle Cavalla
}

\section{To cite this version:}

Agnès Tutin, Iva Novakova, Francis Grossmann, Cristelle Cavalla. Esquisse de typologie des noms d'affect à partir de leurs propriétés combinatoires. Langue française, 2006, Collocations, corpus, dictionnaires, 150, pp.32-49. hal-01098808

\section{HAL Id: hal-01098808 \\ https://hal.science/hal-01098808}

Submitted on 19 Jan 2015

HAL is a multi-disciplinary open access archive for the deposit and dissemination of scientific research documents, whether they are published or not. The documents may come from teaching and research institutions in France or abroad, or from public or private research centers.
L'archive ouverte pluridisciplinaire $\mathbf{H A L}$, est destinée au dépôt et à la diffusion de documents scientifiques de niveau recherche, publiés ou non, émanant des établissements d'enseignement et de recherche français ou étrangers, des laboratoires publics ou privés. 


\author{
Agnès Tutin, Iva Novakova, Francis Grossmann \& Cristelle Cavalla \\ Université Grenoble 3 \\ Laboratoire de Linguistique et Didactique des \\ Langues Étrangères et Maternelles (LIDILEM)
}

\title{
Esquisse de typologie des noms d'affect à partir de leurs propriétés combinatoires
}

\section{INTRODUCTION}

Sans doute pour se démarquer des psychologues, les linguistes ont, dans l'ensemble, accordé davantage d'attention aux verbes de sentiment (voir entre autres Ruwet 1972, 1994, 1995 ; Mathieu 1995, 2000) qu'à leurs homologues nominaux. La catégorie nominale nous paraît cependant couvrir un champ sémantique plus large et présente l'intérêt d'être fréquemument associée à des supports verbaux variés qui pemettent d'en affiner l'étude sémantique, dans la perspective typologique qui est la nôtre ici.

La classe des noms d'alfect ${ }^{1}$ (désormais $N$ affect) regroupe des noms pouvant se combiner avec les supports nooir, ressentir ou épronier et apparaitre en cooccurrence avec le nom sentiment (de). Sémantiquement, ces noms caractérisent un processus psychologique plus que physique et requièrent obligatoirement un actant humain dans le rôle d'agent ou d'expérienceur. Ces critères nous permettent de sélectionner des noms comme anour, orgueil, fristesse et garete, mais de rejeter fam ou soif qui, s'ils se combinent avec les verbes supports retenus, sont plutôt des " noms de sensation " qui apparaissent difficilement avec sentment: "sentment de fom/soif. Nous distinguons donc deux acceptions pour des noms comme souffrnuce ou doulenr qui présentent à la fois un versant psychologique et physique, et ne retenons que la première. Nous excluons également les noms de qualité ou de propriété comme gaieté ou frisfesse dans des contextes comme cette personne est d'nue grande gaietéltristesse où

1. Nous utilisons ce terme comme classifieur générique ( $f$. les "noms psychologiques" d'Anscombre 1095). Flaux \& van de Velde (2000) utilisent également le terme "nom d'afiect", dans un sens légèrement différent ( $f$. section 1 ). 
le nom exprime une propriété attachée à la personne, même si ces noms peuvent être des N_affect dans d'autres acceptions.

Notre étude a pour principal objectif de proposer une typologie des N_affect les plus courants du français en fonction de leurs propriétés combinatoires : les collocations, définies comme les cooccurrences privilégiées de deux constituants linguistiques entretenant une relation sémantique ef syntaxique (cf. Grossmann \& Tutin 2003), et la structure actancielle des noms. Nous considérons que la combinatoire lexicale et syntaxique fournit une trace objective de leurs contenus sémantiques. On peut ainsi associer des dimensions sémantiques à partir de réalisations lexicales et syntaxiques spécifiques. Notre approche permet également de traiter la polysémie des noms d'affect, qui peuvent, selon ces propriétés, appartenir à plusieurs types (multi-appartenance). Elle permet aussi de traiter le cas de lexèmes qui se situent à michemin entre plusieurs types marqués, échappant par là même à une catégorisation stricte. Nous partons d'une première esquisse de typologie, effectuée à partir d'une approche par prototypes. Nous appuyant sur les propriétés dégagées par la combinatoire syntaxique et lexicale, nous proposons une typologie de 40 N_affect retenus.

\section{QUELQUES JALONS ANTÉRIEURS}

Nous laisserons ici de côté les études à orientation cognitive (par exemple, Johnson Laird \& Oatley 1989) et les études linguistiques qui n'ont pas d'objectif typologique (en particulier Leeman 1991, 1995). Anscombre (1995, 1996) distingue les noms de sentiment et d'attitude (Nsa) "endogènes " (ex.: crainte, amour) dont l'origine se confond avec le lieu psychologique - c'est l'individu lui-même qui est à l'origine du sentiment - et les Nisa "exogènes " (ex. : frayeur, agacentent) qui ont une cause extérieure au lieu psychologique. La distinction se fonde sur la structure argumentale, des dérivations morphologiques et quelques configurations lexicales. Cette opposition, bien que pertinente - et reprise ici un peu différemment sous les termes de "nom de sentiment » ( $\approx$ endogène) et "nom d'émotion " ( $\approx$ exogène) -, reste cependant un peu rigide pour rendre compte de certains noms comme respect qui possèdent certaines caractéristiques des deux catégories. Respect a ainsi la structure argumentale d'un nom endogène ( $k$ respect pour qqn) et d'un nom exogène (le respect devant la nature) : il a, à la fois, une source interne et une cause externe. En somme, il nous semble souhaitable d'affiner la bi-partition proposée, pour rendre compte de la complexité des noms d'affect.

Dans l'approche de Flaux \& van de Velde (2000), les noms qui nous intéressent sont des noms abstraits intensits, c'est-à-dire non susceptibles d'avoir une extension temporelle et pouvant être gradués (ex. : the profonde tristesse). Les " noms d'affect » décrivent des émotions et des sentiments qui « sont intérieurs au sujet " contrairement aux états qui leur sont " extérieurs ". Les noms de sentiment (ex. : amour) se distinguent des noms d'émotion (ex. : peur) par le fait que le deuxième actant est obligatoire et que de nombreux noms de sentiment sont dérivés de verbes. Sont rangés dans les noms d'état (et hors de la 
classe des noms d'affect) les noms comme tristesse qui, dans une de leur acceptions, peuvent se combiner avec des séquences comme être en, être dans, vizre dans, sont souvent dérivés de verbes liés à des adjectifs ${ }^{2}$, se pluralisent. La typologie de Flaux et van de Velde, basée sur des critères linguistiques fins, a été une source d'inspiration essentielle pour notre traitement, en particulier la tripolarité entre noms d'émotion, de sentiment et d'état affectif. Le rattachement des états de type affectif à la classe des états ne nous apparaît cependant pas satisfaisante, dans la mesure où elle est en partie contre-intuitive (la tristesse comme état reste un affect) et parce que le critère intériorité/ extériorité est difficile à appliquer.

Buvet et ses collègues (à paraître) proposent une typologie des prédicats d'atfect dans le cadre du modèle des classes d'objets de G. Gross (Gross 1994), en intégrant les prédicats noninaux, verbaux et adjectivaux. 1_es classes sont définies de façon inductive à l'aide d'un ensemble de propriétés linguistiques: structure argumentale (un ou deux arguments), verbes de manifestation physique, constructions syntaxiques spécifiques. Trois hyperclasses sont ensuite créées autour des affects sur la base de propriétés communes : <émotion> (colère, enthousiasme), <sentiment> (nmour, jalousie), <lumeur> (morosité). Cette typologie inductive met en évidence des propriétés linguistiques fines, mais la démarche ne cherche cependant pas à corréler propriétés syntaxiques et sémantiques, objectif que nous avons essayé de poursuivre dans cette étude.

Enfin, mentionnons l'étude approfondie de Mel'čuk \& Wanner (1996) sur les noms d'émotion en allemand qui met en évidence un ensemble de dimensions sémantiques, souvent basées sur des propriétés combinatoires - certaines reprises dans notre étude - permettant d'opposer les noms, sans dresser véritablement de typologie.

Ce trop bref état des lieux montre l'existence de caractéristiques formelles remarquables, liées à la structure actancielle et à la combinatoire lexicale propres aux N_affect. Ces propriétés doivent permettre d'établir une typologie suffisamment fine et souple pour intégrer certains noms qui se laissent a priori difficilement classer.

\section{MÉTHODOLOGIE}

\section{I. Dèmarche}

Pour établir la typologie des N_affect, nous nous fondons sur une étude de corpus effectuée sur Frmitext, à partir de la base catégorisée (tous genres sauf théâtre et poésie, de 1950 à 2005), d'environ 30 millions de mots (420 textes). Notre point de départ est de considérer que les collocations - dans le sens linguistique et non purement statistique du terme (par exemple Tutin \& Grossmann 2002) -, dans lesquelles entrent les noms étudiés, fournissent une base empirique pour décrire les propriétés sémantico-syntaxiques des N_affect. On

2. On a ainsi des séries comme inquiet, inquiéter. intiquít tite. 
peut considérer, en effet, que les collocatifs (ici, essentiellement les collocatifs verbaux et nominaux) fournissent des indications sur les caractéristiques sémantiques des noms avec lesquels ils entrent en cooccurrence. Les propriétés combinatoires ont été établies systématiquement pour tous les $\mathrm{N}$ affect (voir annexe A). Par exemple, la dimension sémantique c contrôle de l'affect s a pu être établie à l'aide de l'observation de la combinatoire lexicale suivante : X (agent/expérienceur) calne, comprime, contient, domine, dompte, exorcise, maitrise, modère, refoule, réfrène, réprime, retient, surnonte, vainc Det N_affect. Ce test montre qu'une telle dimension est pertinente pour angoisse mais inappropriée pour affection, ce type de cooccurrence étant absent du corpus. Notre démarche est donc proche de celle de Blumenthal (ce numéro) qui exploite la notion de "profil combinatoire".

Dans le cas des textes littéraires figurant dans le corpus, les cooccurrents peuvent avoir une valeur stylistique expressive, sortant de l'usage habituel. Nous avons donc éliminé les usages manifestement anomaux. Nous ne nions pas qu'il existe une certaine plasticité sémantique des N_affect, permettant, au moins à certains d'entre eux, d'être typés de manière différente, suivant le cotexte: les collocatifs jouent alors un rôle actif, par exemple en chargeant de dynamicité un nom plutôt statif, comme tendresse?. Il est donc nécessaire de recouper l'ensemble des domnées linguistiques analysées : au bout du compte, il existe bien des tendances permettant d'aboutir à une définition sémantique relativement stable des principaux types, et de situer les lexèmes étudiés par rapport à ces types.

La sélection des noms s'est faite en deux temps : nous avons d'abord établi une liste des 2000 mots les plus fréquents du corpus, puis sélectionné à l'aide des critères définitoires les $N_{\text {_affect les plus tréquents }}{ }^{4}$. Dans un deuxième temps, à l'aide du TLFi, nous les avons désambiguisés en ne retenant que les acceptions pertinentes. Ainsi, pour horrenr, nous avons distingué deux N_affect différents (horreur-peur, et horreur-dégoût) ${ }^{5}$. En fin de compte, notre étude porte sur 40 lexèmes de N_affect ${ }^{\circ}$.

Les noms retenus étant prédicatifs, le traitement devait en priorité analyser leurs structures actancielles, afin de vérifier - et éventuellement d'affiner - les classements élaborés dans la littérature. Cependant, dans notre étude, la structure actancielle des noms ne s'est pas avérée suffisante pour discriminer avec

\section{Par exemple : Une bonffe de tendresse l'enuhit.}

4. Cette étape de sélection précédant la désambiguisation, elle comporte l'inconvénient de ne considérer que la fréquence des vocables et non celle des lexèmes, la seule réellement pertinente. Un travail prenant en compte la fréquence apres désambiguïsation n'était guère envisageable, étant donné le nombre de lexènes à traiter.

5. Nous avons par ailleurs éliminé le nom désii, qui se révélait trop atypique et trop proche d'un nom de sensation, et le nom comnge qui renvoie davantage à une attitude.

6. Adniration, affection, amitie, amour, angoisse, bonher, colère, crainte, dégoht, déscspoir, donleur, ennui, enthonsiasme, estime, élomemont, excitntion, fiente, gêne, haine, honte, horrut (peur), homrur

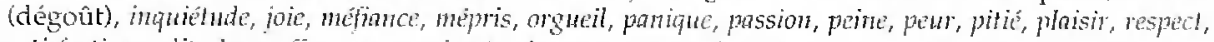
satisfaction, solihude, souffrance, smprise, lendresse, leneur, histesse. 
finesse les noms choisis. Nous avons donc pris en compte un réseau d'autres paramètres sémantiques, établis à l'aide de leur combinatoire lexicale. Outre l'étude de la structure actancielle, nous avons utilisé cinq postes d'observation : l'aspect (phases, ponctuel os duratif), les manifestations de l'affect (cf. la PL Manif, chez Mel'čuk), le contrôle (la manière dont est marquée lexicalement la maîtrise de l'affect par l'expérienceur), la causatioité (inspirer de la haine, causer une gêne) et la "verbalisation " (la capacité qu'a le N_affect d'être objet dans une structure du type dire son/sal N_affect).

D'autres critères ont été écartés, parce qu'ils nous semblaient moins directement nécessaires dans l'optique typologique qui était la nôtre, ou qu'ils semblaient impraticables : l'étude de la détermination des $N$ affect - parce qu'elle aurait demandé une investigation à part entière-, l'emploi des verbes supports standard a priori trop peu contraint, l'étude des collocatifs adjectivaux qui aurait été intéressante si nous avions pris en compte la question de l'intensitéz, et la prise en compte des dérivations morphologiques qui ne nous a pas semblé fournir un critère fiable, étant donné les modifications de sens qu'elle entraîne fréquemment.

\subsection{Prototypes des $\mathbf{N} \_$affect}

Pour éviter à notre démarche une indésirable circularité (on définit les N_affect à l'aide de propriétés linguistiques qui, à leur tour, servent à dresser une typologie des $N_{\text {_affect}}$, nous postulons, en prenant en compte les travaux précédents, l'existence de trois catégories principales de N_affect (respectivement $N$ sent, $N$ émotion, $N$ _état_affect) incarmées par des prototypes nominaux, qui en sont les " meilleurs exemplaires".

\subsubsection{Les prototypes du N_sent: affection, amitié}

Le premier type mis en évidence ( $N_{-}$sent), incarné par les exemplaires affection et nmitie, renvoie à une relation interpersonnelle qui implique deux actants sémantiques humains: l'affection/l'amitié d'une personne $X$ pour/enters une personne $Y$. Les deux actants ne sont pas nécessairement réalisés en surface, mais sont toujours sous-jacents. Le premier actant sémantique est davantage un agent qu'un expérienceur : il est "actif " et participe à la construction de l'affect. Le second actant est un objet sémantique, plus qu'une cause. Sur le plan aspectuel, les N_sent ont une certaine stabilité : ils sont plutôt duratifs. Ils connaissent également des phases (le début, la continuation, la fin).

Si l'on observe la combinatoire lexicale, on remarque que les $N$ sent prototypiques sont rarement accompagnés de collocatifs exprimant le contrôle (maitriser, dominer, retenir) ou des verbes qui expriment la manifestation physique, comme bondir, crier ou trembler ou l'effet sur l'individu (ex. : envahri). Par ailleurs, les $N_{-}$sent prototypiques sont rarement associés à des

7. Nous avions jugé - à tort, cf. conclusion - que l'intensité n'était pas un critère déterminant dans notre perspective typologicue. 
verbes causatifs comme susciter, causer dans la mesure où le destinataire du N_sent - la personne vers qui se dirige le sentiment - n'est pas véritablement une cause, mais plutôt un objet. Enfin, ils se combinent avec des verbes de parole qui impliquent une forme de communication entre les deux actants (dire son nmitié) plutôt que l'expression "émotive» (crier son désespoir).

\subsubsection{Les prototypes du N_émotion : surprise, peur (sens ponctuel) ${ }^{8}$, angoisse (sens ponctuel)}

Les $N$ émotion prototypiques sont des $N$ _affect réactifs?. Le premier actant s'apparente plus à un « siège » ou à un "expérienceur » qu'à un agent. Le second actant sémantique, facultatif en surface, n'est pas toujours sousjacent: il peut y avoir une angoisse, même ponctuelle, sans cause déterminée. Ce deuxiène actant, souvent introduit par deonnt, du fait de, à cause de, est une cause et non un objet.

Les N_émotion prototypiques sont préférentiellement ponctuels, et si plusieurs phases peuvent apparaitre pour ce type de N_affect, l'inchoatif est le plus productif (I'angoisse surgit, la peur le saisit, ...). La combinatoire lexicale de surprise et de peur montre que les verbes de contrôle sont fréquents (donmer, mâ̂triser, sumonter), ainsi que les verbes de manifestation (trembler) hurler/reculer de peur/d'angoisso) et les verbes causatifs. Les verbalisations sont plus « expressives» (hurler sa peur, crier son angoisse) que communicatives.

\subsubsection{Les prototypes du N_état_affect : bonheur, ennui, solitude}

Dans cette troisième catégorie, les prototypes ont un, ou parfois deux actants. Le premier actant, humain, est l'expérienceur. Le second actant est facultatif ou inexistant (comme pour solitute). S'il apparaît, c'est une cause (ex. : lo bonlicm de wive).

Sur le plan aspectuel, les N_état_affect prototypiques sont duratifs (vivre dans la solitude, mger dans li bomheur). Leur caractère plus ou moins permanent les prédispose moins au contrôle. Plus intérieurs que les $N$ _émotion, ils sont moins susceptibles d'être associés à des manifestations plyysiques. Comme ils ne sont pas nécessairement liés à une cause, les causatifs sont a priori moins fréquents.

Il nous reste à présent à vérifier dans quelle mesure cette première typologie peut s'appliquer à l'ensemble des N_affect sélectionnés, et éventuellement à identifier des types intermédiaires. L'étude de corpus a pour fonction, non seu]ement de valider ou d'invalider la typologie esquissée à partir des prototypes. mais aussi de la compléter et de l'affiner. Nous analyserons donc, en nous appuyant sur le corpus, les propriétés combinatoires spécifiques de chaque nom. en les confrontant à celles qui ont été établies à partir des prototypes.

8. La précision " sens ponctuel " renvoie à une acception de peur qui a un aspect ponctuel (la peru"

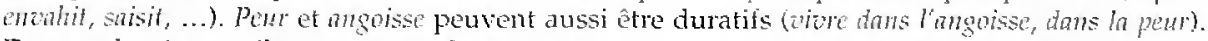
Dans ce ternier cas, ils seraient proches du prototype des N_état_atfect.

9. Cf. Anscombre (1905: 47) : 11 lle nom exogènel est en quelque sorte une rénction à un événement externe." 


\section{COMBINATOIRE DES N_AFFECT}

\section{I. Les structures actancielles}

Nous avons d'abord recensé les différentes réalisations en surface des actants que régissent nos $\mathbf{N}$ a affect. Nous les avons, par la suite, corrélé à des rôles sémantiques différents: agent, expérienceur, objet, cause. Tout en nous inspirant du classement sémantique des actants de Mel'čuk $(1995,1996)$, nous avons essayé de l'affiner ${ }^{10}$ en les caractérisant à l'aide de rôles sémantiques afin de bien distinguer objet et cause du N_affect.

Nous avons également été vigilants au fait qu'un actant sémantique sousjacent n'implique pas forcément son instanciation syntaxique, ce qui rend les structures actancielles sémantiques et syntaxiques correspondant à un N_affect non isomorphes. Nous avons dégagé cinq structures actancielles:

a) N_affect de personne $X$ [agent] pourlenvers/à l'égard de personne $Y$ lobjet]

C'est la structure actancielle relevée pour les N_sent prototypiques amitié, affection. Ce schéma rend compte du caractère humain des deux actants du N_affect interpersomnel. L'étude de corpus nous montre que d'autres N_affect conme anour, haine, tendresse peuvent avoir cette même structure actancielle.

\section{b) N_affect $d e$ personne $X$ [agent] de/pour $Y$ non humain [objet]}

Anour, haine, tendresse peuvent aussi apparaître dans ce schéma qui se distingue du précédent par le trait non humain du $2^{2}$ actant dont la réalisation syntaxique est un $\mathrm{SN}$ et ne peut être une construction infinitive ou complétive: par exemple, l'amour/la hame des voynges, la tendresse pour la nature. (Sagan).

\section{c) N_affect de personne X lagent] envers/à l'égard de personne $Y$ [objet] ì cause de $\mathrm{Z}$ [cause]}

Par ailleurs, il existe aussi une structure à trois actants, signalée dans le DEC (Mel'cuk et al. 1984, 1992) : l'admiration de Pierre envers Jacques pour son courage. lci, en plus des deux actants humains, apparaît un $3^{\mathrm{e}}$ actant $(Z)$ qui est la cause du N_affect.

Or, nous n'avons pas trouvé (dans Frontext) des exemples de ce type qui réalisent les trois actants à la fois, ce qui nous amène à considérer que la réalisation intégrale de cette structure en surface n'est pas possible ou, en tout cas, très peu probable. Autrement dit, le $3^{2}$ actant cause (pour, à conse de son courage), même s'il est sémantiquement sous-jacent, n'est jamais réalisé syntaxiquement en cooccurrence avec le second. Nous concluons qu'à la différence des verbes ${ }^{11}$, les $N$ _affect ne réalisent pas les trois actants à la fois, ce qui est probablement dû à la complexité de la structure du syntagme nominal.

\footnotetext{
10. Nous avons remarqué que parfois l'actant $Y$ ', bien que détini comme objet ou somrce de l'alfect, pourait en être aussi la comse qui comespond nomalement à l'actant Z (Mel'suk \& Wamer 1996).

11. Par exemple: Paul míprise le ministre pour son hyporisie.
} 


\section{d) N_affect de personne X lagent] ou [siège] à cause de/deonant/ì la aue de $\mathrm{Z}$ [cause], réalisé comune $\mathrm{SN} / \mathrm{V}$ inf/Complétive}

Dans ce schéma, le $2^{e}$ actant [cause] peut avoir des réalisations syntaxiques différentes: SN prépositionnel (de, du fait de, devant $\mathrm{N}$ humain ou non humain), V inf ou subordonnées complétives. Il correspond aux N_affect qui se rapprochent des prototypes des émotions. C'est le cas de crainte (cf. la crainte du scandale, du directeur/de le réveiller, qu'il parte) mais aussi de peur, d'enthousiasme ${ }^{12}$, de plaisir, de surprise.

Certains N_affect, en plus des réalisations syntaxiques du $2^{e}$ actant décrites ci-dessus, peuvent se combiner avec un autre actant humain, qui a plutôt le rôle de source/objet que de cause de l'affect (cf. sa colère contre pierre, sa honte vis-à-चis de ses amis, sa gêne/son dégoût envers son nuari). Ces noms présentent une structure actancielle qui est proche de celle du schéma $c$.

\section{e) N_affect $d e$ personne $X$ [siège]}

On a également recensé un schéma à un actant qui correspond aux N_état_affect prototypiques : solitude ou emmui. Leur actant unique est le siège de l'affect.

Pour résumer, les prototypes des N_état_affect (ennui, solitude) ont une structure uniactancielle.

Les noms à structures biactancielles se distinguent par le rôle sémantique du $2^{e}$ actant : [objet] pour les $\mathrm{N}$ sent prototypiques (affection, amitié) ou [cause]

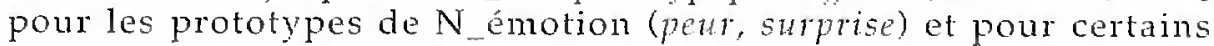
N état_affect (bonheur, désespoir, sonffrance), qui se rapprochent des N_émotion.

Enfin, il existe des N_affect à structures triactancielles, où le $3^{e}$ actant $n^{\prime}$ est jamais réalisé en cooccurrence avec le $2^{2}$ actant. Deux cas de figure se présentent ici, toujours selon le rôle sémantique du $3^{e}$ actant : celui-ci peut être [cause] pour les $\mathrm{N}$ sent comme respect, admiration ou mépris ; ou [objet] comme pour honte, colère, gêne.

Le critère actanciel, bien que primordial, ne s'avère pas toujours discriminant pour notre typologie des $\mathrm{N}$ aftect. Par rapport aux prototypes utilisés, il montre néanmoins l'existence de types intermédiaires de $N$ sent se rapprochant des N_émotion, car causés (ex. : admiration, mépris, respect), et de N_émotion proches des N_sent, car ayant une dimension interpersonnelle (ex. : honte, colère, gêne).

\subsection{L'aspect des $N_{\text {_affect }}$}

\subsubsection{Phasiques vs non phasiques}

L'aspect phasique oppose des N_affect marquant les phases à des N_affect les marquant peu. De manière générale, les noms proches des prototypes des N_état_affect, comme bonhewr ou solitude, sont peu marqués du point de vue

12. On a ceprndant relevé dans Frantext enthonsiasme pour le gétún qui semble présenter une métonymie suggérée par le contexte : pon $k$ gérichl = pour ses exploits, son comportement. 
des phases. C'est le cas également des N_sent, comme annitié et affection, avec quelques nuances: si le nom tendresse connaît peu de collocations traduisant les phases initiales et terminales, on le rencontre cependant dans certaines structures binominales à valeur inchoative (une bouffée de tendresse l'enohit). Amour ou haine apparaissent plus phasiques qu'amitié ou affection, et se rapprochent sur ce plan des $N_{-}$émotion. À l'inverse, les noms impliquant une évaluation, comme mépris ou estime, sont peu sensibles à la dimension phasique: la plupart du temps, ils se rencontrent dans des constructions à verbe support, ou causatives, non marquées aspectuellement (nvoir de l'estime, susciter le mépris), ou bien encore les associant aux manifestations (nn somire de mépris). À ces types peu phasiques, on peut opposer les noms, qui, comme les prototypes de nos N_émotion, sont pleinement phasiques: $c^{\prime}$ est le cas de colère, par exemple. L'inchoatif de déclenchement est typiquement signalé à travers les collocations liées aux verbes supports (se mettre en coleve). Colère combine par ailleurs inchoatif et ponctuel, ce qui explique son emploi avec certains collocatifs ( $c f$. Blumenthal, ce numéro) : l'affect est mis en relation avec l'agent humain, qui peut éventuellement le contrôler, mais qui le subit généralement (ćt. la colère l'enoahit).

\subsubsection{Ponctuels vs duratifs}

Si l'on suit le modèle fourni par les prototypes, la combinaison fréquente entre aspect phasique inchoatif et aspect ponctuel caractérise en effet les noms qui se rapprochent des prototypes des $N$ émotion, comme le montre leur emploi dans les collocations binominales du type acces de + N. Flambéc apparaît avec colère et passion; ćlan avec tendresse, enthousinsme, joic, colère, fierté, amour, tendresse; échir avec joie; explosion avec joie, colere et, plus surprenant, tendresse; accis avec désespoir, haine, colere, angoisse. Joie et admiration sont les seuls à apparaître avec transport(5). Dans les structures binominales du type explosion de $+\mathrm{N}$, élan de $+\mathrm{N}$, échir de $+\mathrm{N}$, transport de $+\mathrm{N}$, l'aspect ponctuel se combine cependant avec des valeurs sémantiques plus spécifiques. Ainsi, ćlan apparaît dans le corpus comme plus typiquement associé à des noms de polarité positive (nffection, amour, tendresse), tandis quaccès, ou crise, sont plutôt associés à des noms qui se prêtent à une interprétation pathologique, comme colère et désespoir - même si l'on trouve aussi des emplois moins habituels (un accès de joie, rencontré une fois).

L'examen des structures binominales comportant les marqueurs ponctuels accis et álan permet de distinguer des noms qui admettent le ponctuel ou le duratif, mais rarement les deux - en dehors d'enthousiasme -, d'autres qui les admettent mais à une fréquence moindre (peur, tristesse, honte, anitié, haine, passion, ennni, panique, surprise), d'autres, enfin, qui semblent les exclure, au moins dans un emploi standard (satisfaction, peine, étonmenent, estime, mépris). Bonffées classifie aisément plaisir, honte, cotere, haine, et semble par ailleurs assez tolérant (des bouffées de bouhrur). L'examen des classifieurs ponctuels dans les structures bi-nominales témoigne de peu d'exclusions complètes. Certains noms, comme par exemple satisfaction et estime, apparaissent quand même difficiles à " ponctualiser ». 
Si les N_émotion typiques (surprise, angoisse) sont, comme attendus, ponctuels, certains noms, classés intuitivement comme $N$ sent (nnour, hanc, joie), peuvent être aussi bien ponctuels que duratifs, ce qui est aussi en accord avec leur possibilité de se combiner avec les trois phases. Impliquant une certaine stabilité de la situation référentielle (Desclés 1994), ils ont cependant une tendance au duratif. À l'inverse, peur, intuitivement classé comme N_émotion, apparaît parfois dans des emplois duratifs, ce qui le rapproche alors des N_état_affect. En ce qui concerne les N_état_affect, même si, par définition, ils sont duratifs, il est intéressant de noter qu'ils peuvent également évoluer du statif au dynamique : cela explique les combinaisons possibles de certains d'entre eux dans des collocations impliquant le ponctuel (crise de desespoir, être enuhi par le désespoir vs être en plein désespoir, viore dans le désespoir), ce qui les rapproche des N_émotion.

\subsection{Les manifestations physiques extérieures}

De nombreux $N$ affect sont décrits comme ayant une composante sémantique liée à une manifestation physique extérieure de l'agent/expérienceur atteint par l'affect (Mel'cuk \& Wanner 1996; Buvet et al. à paraître), qui se traduit sur le plan lexical par des cooccurrents verbaux (on bondit de joie, recule d'effroi) ou nominaux (on pousse un cri de peur, un hulement d'effroi). Du fait de leur extrême diversité, il nous a semblé indispensable de différencier les types de manifestation afin de dégager les caractéristiques des N_affect.

\subsection{Les expressions du visage}

De nombreux collocatifs permettent de préciser la façon dont l'afiect se reflète sur le visage de l'agent humain : un regand d'adnination, une expression de haine. Le nom regard exprime une directionnalité qui le prédispose à avoir des personnes comme objet sémantique et, de ce fait, à apparaître plutôt avec des noms proches des $N$ sent, mais, comme le nom expression, il exprime le sentiment quand il accompagne des noms comme crante ou dégoût.

Un deuxième ensemble d'expressions porte plus spécifiquement sur la complexion du visage de l'agent impliqué dans l'affect, toutes liées à des affects assez intenses : blêmir de rage, rougir de colèrelplnisir, pâlir de peur. Tel n'est pas le cas des expressions plus métaphoriques semblant refléter à l'extérieur l'état intérieur de manière globale, sans que la manifestation se localise sur un organe précis, comme dans rayonner/irndier de bontent, exulter de fierté, resplendir de joie. Ces collocatifs sont liés, pour la plupart, à des affects de polarité positive.

\subsubsection{Les manifestations physiques « subies» et « actives»}

Les manifestations physiques "subies" sont des expressions comme suer d'angoisse, trenbler de peur, sécanonir de terrent qui répondent au schéma syntaxique $V$ de $N$ affect (cf. Leeman 1991), où de $N$ est un complément de cause du verbe. Nous écartons ici les expressions verbales (crier, hurler) qui seront détaillées plus loin. Ces manifestations sont réactives et ne sont pas contrôlées par la volonté de l'agent/expérienceur qui les subit. Bien entendu, 
certaines de ces expressions comme défaillir d'amour ne doivent pas être interprétées de façon littérale et ont souvent aussi une valeur métaphorique qui exprime indirectement l'intensité de l'affect éprouvé. De manière générale, si la cooccurrence avec des affects agréables (frémir de joie) et peu intenses (bâiller d'cmmi) est courante, les manifestations invalidantes (trembler, suffoquer, défaillit, ...) sont particulièrement fréquentes avec des affects intenses et négatifs.

Contrairement aux expressions précédentes, les manifestations pliysiques "actives" supposent une décision volontaire de l'agent qui est ici plus actif que réactif. Ce sont essentiellement des verbes, plus marginalement des expressions comme élan ou geste, dans leur interprétation littérale. Les verbes de manifestation physique - bondir, sauter, reculer, éclater - sont généralement liés à des noms d'affect intense comme joic, colère, peur.

\subsubsection{Les effets de l'émotion sur l'agent}

Une série de collocatifs verbaux qui a le nom d'affect comme sujet et l'agent comme objet direct (la peur paralyse qqu, la colère conoulse, il csi frappél saisi d'étonnement, être cloué de panique, ...) s'interprète comme le résultat d'un effet violent de l'affect sur la personne ( $c$. la dimension "possession " utilisée par Goossens (à paraître) dans sa typologie des noms de sentiment). Nombre de ces verbes ont une variante causative (cf. Balibar-Mbrati 1995, p. ex.: L'angoisse le paralyse (Quelque chose le parnlyse d'angoisse)). Là encore, ces collocatifs sont plus fréquents avec les noms d'affect intense (angoisse, horrent, peur, terreur) et sélectionnent préférentiellement des noms de polarité négative bien que admiration, amonr et passion les admettent également.

\subsubsection{Les manifestations verbales}

Nous regroupons dans cette catégorie des lexèmes qui extériorisent verbalement l'émotion, sans communiquer véritablement de contenu : structures nominales du type cri, hurlenent, gémissement, ... de $\mathrm{N}$ affect ou structures verbales $V$ de $N$ affect (hurler, génir, crier). Ces manifestations sont fréquentes dans le corpus avec les émotions intenses négatives comme angoisse, colère, peur, temeur, possibles avec joie, surprise, mais rares avec des affects interpersomnels intenses comme admiration.

En conclusion, deux pôles clairement marqués se dégagent :

- Les N_affect comportant pas ou peu de manifestation (comme crninte, estinne, orgneil, ennui, amitié, peine) renvoient aux prototypes de N_état_affect et de N_sent.

- Les N_affect comportant tous les types de manifestation (colere, horrut (peur), peur, panique, surprise, angoisse, enthousiasme) sont majoritairement des noms d'affect négatif intenses et, ayant une composante aspectuelle ponctuelle, proches du prototype de N_émotion.

Entre ces deux pôles, il apparaît difficile de dégager des sous-types clairement identifiables. Les affects peu intenses et de polarité positive tendent à produire peu d'expressions de manifestation; il est cependant clair que la dimension : manifestation > est corrélée à deux dimensions que nous avions 
jugées a priori non pertinentes pour notre objectif typologique : $l^{\prime}$; intensité , et la 'polarité .

\subsection{Le contrôle}

Comme les manifestations physiques, le fait de "perdre le contrôle " est souvent invoqué comme trait caractéristique pour les émotions. On peut ici faire l'hypothèse que les noms proches de nos prototypes des N_émotion sont davantage sensibles à cette dimension puisque la cause est extérieure à l'agent/expérienceur, que c'est en quelque sorte l'émotion qui agit sur l'individu qui, par réaction, veut en limiter les effets ou les manitestations extérieures. En revanche, les noms proches des prototypes des N_sent deviaient, eux, avoir une combinatoire pauvre en verbes de contrôle puisque l'origine de l'affect est l'agent lui-même (caractère «endogène"). Les N_état_affect, plus duratifs, devraient également comporter peu de collocatifs de ce type.

De nombreux verbes expriment le contrôle de l'individu sur l'émotion : l'individu (a) « apprivoise » l'émotion (maftriser, dominer, dompter, exorciser sa peur), (b) l'éradique (refouler, vaincre, sumnnter sa peur), (c) en modère les effets (calmer, modérer son angoisse), (d) l'empêche d'apparaître (retenir, contenir, réfréner sa colère). Le contrôle s'exprime également dans la manifestation de l'émotion ${ }^{13}$ : dissimuler, taire son mépris, sa peine.

L'observation des collocatifs de contrôle associés aux noms pernet de dégager certaines tendances.

Les noms qui se combinent avec les deux types de collocatifs de c contrôle > (contrôle de l'affect et de sa manifestation) - angoisse, dégoût, enmi, possion, prine, surprise, ... - sont, de façon peu surprenante, majoritairement de polarité négative (10 sur 13) et intenses. Dans cette classe, apparaissent deux des N_émotion prototypiques comme angroisse ou peur, mais également des $\mathrm{N}$ état_affect comme enmi ou peine. On n'observe cependant ici aucun $N$ sent typique.

A l'inverse, certains noms n'ont aucun collocatif de contrôle. Ils forment apparemment une classe hétérogène. Certains peuvent avoir un objet sémantique humain (annitié, nućfinnce, tendresse), alors que d'autres ont un deuxième actant facultatif ou inexistant (solitude, tristesse, bonheur). La plupart ont une intensité faible et peuvent être duratifs, hormis panique et terreur qui, à l'inverse, sont ponctuels et si intenses qu'aucun contrôle n'apparaît ici envisageable.

Les noms accompagnés de collocatifs exprimant seulement le contrôle de la manifestation - amour, étonnement, mépris, pitié - sont peu intenses, comportent majoritairement un objet vers lequel le contrôle de la manifestation est dirigé, et sont donc préférentiellement liés aux $N$ sent prototypiques.

Les noms avec contrôle de l'émotion, mais pas de la manifestation - colère, crainte, désespoir, horreur (peur), joie - sont plutôt intenses, et la plupart sont perçus spontanément comme des N_émotion.

13. C) Mel'suk, Clas \& l’olguère (1995) qui distinguent le contrôle de l'émotion même (mâtriser, tominer) de celui de sa manifestation extérieme (chdher, dissmuly, inire). 
Pour résumer, comme pour les manifestations, le caractère plus ou moins intense de l'affect semble jouer un rôle déteminant. Ainsi, les N_affect apparaissant avec des expressions du contrôle sont proches des types de $\mathrm{N}$ émotion et N_état_affect intense. Les N_affect qui n'en comportent pas sont souvent peu intenses ou si intenses (panique, terreur) que le contrôle est impossible.

\subsection{La causativité}

Parmi les collocatifs verhaux associés aux N_affect étudiés, nous avoms dégagé trois sous-ensembles de verbes causatifs :

(a) des causatifs que nous avons appelés "neutres » faire, provoquer, inspirer, causer + Det N affect,

(b) des causatifs dits «intensifs", regroupant aussi bien des verbes qui renforcent l'affect comme renforcer, atgmenter, attiser, que ceux qui l'apaisent: apaiser, calmer + Det N_affect;

(c) des causatifs "phasiques" correspondant aux phases initiale, médiane on finale de l'affect : engendrer, déchencher, entretenir, chusser, dissiper + Det N_affect.

Notre hypothèse, à savoir que les N_émotion devraient apparaître plus souvent accompagnés de causatifs neutres, car toujour's causés, s'est globalement confirmée. Les $N$ émotion prototypiques (peur, smprise) se combinent aisément avec causer, provoquer. C'est également le cas de crante, colert, enthousiasme, honf. En revanche, les noms proches des N_sent (nffotion ou anitib) ne se combinent que très rarement avec des causatifs neutres, ce qui peut être lié à leur caractère interpersonnel. Par exemple, provonuer la ficrté ou inspirer la haine n'apparaissent que deux fois dans le corpus. Enfin, les N_affect comme bonhur, doulcur, cmmi peuvent également être provoqués; ce trait ne permettant donc pas de distinguer le type des N_émotion de celui des N_état_affect.

Pour ce qui est des verbes causatifs qui expriment en plus l'intensité (exciter, augnenter, renforcer la pent, la crante, In colire) ou l'apaisement (les calmer ou apriser), là encore, ce sont les noms du type N_émotion qui les appellent le plus fréquemment. Cette caractéristique intensive apparaît liée à celle des manifestations actives $*$ (bondir, sunfr), ou des verbalisations "émotives" (crier, mmer). Contrairement aux N_émotion, les N_état_atfect n'ont que rarement pour collocatifs des verbes causatifs intensifs. Les noms prototypiques bonhlur et ennni ne présentent pas de telles occurrences.

Enfin, les verbes causatifs "phasiques » ne peuvent être considérés comme discriminatoires pour nos tyes. Toutefois, des tendances se dessinent ici aussi, à savoir que les prototypes des N_état_affect bonhour et emnui se combinent souvent avec la phase finale (chasser, tissiper, briser). Les noms proches du type N_émotion apparaissent le plus souvent avec les verbes de phases initiales (engendrer, déchencher, réveiller la perr, la cminte, la colère, ha joie) et, plus rarement, avec ceux indiquant "la sortie causée " de l'émotion (briser la purr, dissiper lo crninte). Cette tendance est contirmée par les cas fréquents de verbes inchoatifs avec les émotions. Enfin, les $N$ sent apparaissent tendanciellement avec des causatifs indiquant la phase intermédiaire : entretenit, nnintenir frmitié. l'amonr, les aulres phases étant statistiquement très peu représentées. 


\subsection{Les verbalisations}

Nous nous intéressons ici aux verbes de "dire ", de structure $V$ son/sa N_affect, qui indiquent le mode de verbalisation utilisé : exprimer, dire, déclarer, hunler sa colère. Certains noms n'appellent pas ce type de verbalisation : on ne déclare, dit, n'exprime pas (ou peu) son orgueil, mais la majorité des N_affect l'admet. Ces collocations sont rarement métaphoriques, bien qu'on rencontre marginalement vomir sa haine ou déverser sa colete. En examinant le corpus, nous constatons que les collocatifs verbaux se divisent nettement en deux classes sémantiques que nous avons appelées "émotives " et " communicatives".

\subsubsection{Les verbalisations «émotives»}

Ce champ rassemble des collocations dans lesquelles l'échange communicationnel avec le deuxième actant n'est pas forcément sollicité : hurler son désespoir n'appelle pas une réaction de l'interlocuteur, l'échange n'est pas attendu. Ces réactions sont peu contrôlées par l'agent, mais sont dirigées vers un interlocuteur (contrairement aux manifestations verbales comme hurler de colere ou crier de doulcur).

Les collocatifs de "verbalisation émotive "sont fréquents avec des noms proches du prototype des N_émotion, mais aussi de N_sent intenses.

\subsubsection{Les verbalisations « communicatives»}

Il s'agit de verbalisations que le locuteur contrôle davantage, dans lesquelles l'échange a pour thème l'affect ressenti. Les verbes utilisés dénotent une intention (cf. l'intentionnalité chez Ruwet 1994) de communication (confier, partager, exprimer, ténoigner). Ces collocations apparaissent majoritairement avec des noms proches des N_sent et des N_état_affect (lánoigner son affection, témoigner son enthousizsme; on ne témoigne pas sa peur ou se surprise). On relève aussi quelques N_émotion associés à des verbalisations communicatives, mais plus rarement : on cxprine sa crainte, on partage our on témoigne sa joie, on épanche ou on confie sa peine...

Pour conclure, le recours à la polarité s'avère indispensable si l'on veut se repérer dans le champ des verbes de "dire ". Les verbalisations intenses (hurler, crier) apparaissent essentiellement avec des noms proches des prototypes des N émotion à polarité négative, alors que les verbalisations «communicatives » (dire, aroher) s'associent préférentiellement à des noms des types $N$ _sent et $N$ _état_affect.

\section{VERS UNE TYPOLOGIE DES N_AFFECT}

Les résultats de nos analyses précédentes nous conduisent, pour établir notre typologie, à retenir parmi les 23 traits étudiés, les propriétés les plus discriminantes. Nous avons sélectionné trois critères principaux : la structure actancielle, l'opposition aspectuelle duratif is ponctuel et le contrôle, ainsi que deux critères secondaires: manifestation et ierbalisation. Les autres paramètres (phases du procès et causntivitê ne sont pas repris dans l'état final de notre typologie. 
On constate que la plupart des N_affect examinés s'écartent des trois prototypes postulés ( $N$ _sent, $N$ émotion, $N$ _état_affect) dans la mesure où ils ne partagent qu'un sous-ensemble de propriétés ou ont des propriétés communes à plusieurs types. Il est nécessaire de passer d'une logique de prototypes à une logique de classes: les propriétés combinatoires ont été analysées pour établir les types, en dégroupant les acceptions des noms polysémiques. Par exemple, peur et angoisse se rapprochent des N état affect con vit dans la peur, dans l'angoisse), mais également des N_émotion par leur caractère ponctuel (crise d'angoisse, accès de peur).

\section{I. Classes de noms d'affect}

Notre typologie intègre à présent un sous-ensemble de critères combinatoires, reposant sur des conditions nécessaires et suffisantes. On aboutit à un classement composé de six classes:

a) C1 \& noms d'affect interpersonneIs , anitié, affection, annour, tendresse, hainet regroupe les noms comportant deux actants; le second actant est un objet sémantique toujours sous-jacent (mais parfois non réalisé syntaxiquement) qui est préférentiellement humain ${ }^{14}$. Les noms sont durables et ne se "contrôlent " pas.

b) C2 ، noms d'affect interpersonnels causés , frespect, mépris, estime, méfinnce, admination, pitié se distingue de C1 par le tait qu'elle regroupe des noms qui ont un actant supplémentaire (au moins sous-jacent), qui a un rôle sémantique de cause. Ces N affect ont généralement une dimension sociale, normative. Us sont durables ef ne sont pas accompagnés de verbes indiquant le contrôle. Les collocatifs verbaux ne marquent pas les manifestations physiques subies ni l'expressivité (comme humler). On les rencontre, en revanche, avec des verbes de communication comme exprimer, commumiquerson N_affect.

c) C3 4 noms d'affect ponctuels réactifs > isurprise, peur, angoisse, joic, excitation, horren (peur), désespoir, enthousiasmi, sonffrance, panique ef terren? regroupe des noms possédant un deuxième actant, qui a un rôle sémantique de cause. Ils sont plutôt ponctuels, comportent des verbes de manifestations subies et s'associent à des verbes expressif́s.

d) C4 r noms d'affect interpersonnels réactifs , lcolère, honte, dégout, furreur (dégoût), gêne, inquichtude) intègre des noms qui ont un actant au rôle sémantique de cause, mais aussi, souvent de façon sous-jacente, un objet humain. Ce ne sont pas de purs affects: ils ont également une dimension évaluative et morale. Ils peuvent être ponctuels et comportent des verbes de contrôle, de manifestations physiques subies et de verbalisation expressive.

e) C5 ، noms d'affect duratifs non contrôlés , \{ennui, bonheur, solitude, plaisit, orgueil, satisfaction, trislesset. Leur deuxième actant, la cause, est facultatif.

14. Les emplois avec 2 actant $\mid$ - humain] sont statistiquement moins fréquents pour être pris en considération dans cette classe. 
Ils sont durables et ne sont pas associés à des collocatifs exprimant le contrôle.

f) C6 s noms d'affect duratifs contrôlés > \{peine, crainte, angoisse, désespoir, douleur, fierté, horteur (peur), joie, peur\} comporte des noms ayant la même structure actancielle qu'en $\mathrm{C} 3$, mais pouvant être duratifs, et souvent accompagnés de verbes de contrôle. Ces $N$ affect sont par ailleurs souvent intenses.

À l'issue de cette typologie, quelques $N$ affect demeurent inclassables. C'est en particulier le cas de possion, qu'on peut caractériser comme un < nom d'affect interpersonnel de très grande intensité, se rapprochant d'un $N$ affect de $C 3$ par la présence de verbes de contrôle et de nombreuses manifestations. Étonnement se révèle également atypique. Bien que ponctuel comme les éléments de $C_{3}$, il n'en a ni la combinatoire de contrôle, ni les manifestations. C'est aussi un des rares $N$ affect de notre corpus avec surprise à ne pas présenter une polariłé marquée (il n’est ni positif, ni négatit). Par ailleurs, une certaine redondance s'observe entre $\mathrm{C} 3$ et $\mathrm{C} 6$, de nombreux N_affect comme pen ou joie appartenant simultanément aux deux classes. Fusionner ces deux classes ne nous semble cependant pas souhaitable, puisque la polysémie des $\mathrm{N}$ appartenant aux deux classes n'est pas complètement systématique. Par exemple, sumprise est exclusivement ponctuel, alors que peine est plutôt duratif.

Bien entendu, les classes comportent des éléments hétérogènes que l'on pourrait encore subdiviser. Ainsi, on poumait au sein de C 1 distinguer les N_affect à objet exclusivement humain (affection, amitié) et ceux qui présentent moins de contraintes sélectionnelles sur leur objet comme anour ou haine. Il serait également possible, en étudiant des propriétés non définitoires mais fréquemment associées aux éléments de la classe, de déterminer le degré de typicité d'un N_affect domné dans sa classe. Par exemple, dans C3, les noms terrenr et panique sont les seuls à ne pas s'associer à des verbes de contrôle du fait de leur intensité extrême.

\subsection{Comparaison avec une classification automatique}

À titre exploratoire, parce qu'il est difficile de manipuler manuellement simultanément un grand nombre de propriétés, nous avons comparé notre classification avec un regroupenent établi autounatiquement à partir de nos propriétés linguistiques. Ce traitement a été effectué par Gilles Bisson et Benoît Lemaire ${ }^{15}$ du laboratoire Leibniz de l'INPG de Grenoble, en utilisant une classification ascendante avec distance euclidienne prenant en compte nos 23 propriétés (booléennes) ${ }^{\text {th }}$ sans pondération spécifique. Les résultats de cette classification confirment les classes proches des prototypes: classe $C 4$ des $N$ affect ponctuels et réactifs (proche des $N$ _émotion), classe $C 1$ des

15. Nous remercions chaleureusement nos collègues pour cette collaboration.

16. Un " + " ou un " - sont associés à la propriété, selon qu' elle se vérifie ou non pour le mom considéré. 
N_affect interpersonnels (proche des N_sent), classe C5 des N_affect duratifs non contrôlés (proche des N_état_affect). En revanche, les autres classes, moins immédiates, ont été dispersées dans le classement. On relève que deux de ces classes intermédiaires ( $\mathrm{C} 2$ et $\mathrm{C} 4$ ) comportent une dimension sociale et axiologique. Ces deux aspects ne semblent pas se tratuire clairement dans la combinatoire lexicale examinée avec la méthode de classification automatique. Peut-être aurait-il par ailleurs fallu pondérer davantage les propriétés considérées comme essentielles (structure actancielle, caractère ponctuel/ duratif et contrôle) pour retrouver les classes établies manuellement?

\section{CONCLUSION}

À l'issue de cette étude, on constate que la méthode proposée - l'étude de la combinatoire syntaxique et lexicale dans une perspective typologiquesemble tenir ses promesses. Elle permet d'établir des profils sémantiques à partir de propriétés formelles, moins contestables que les traits sémantiques dégagés à l'aide de la pure intuition sémantique. Le champ sémantique des noms d'affect peut ainsi être subdivisé à partir de plusieurs propriétés établies par la combinatoire. Seul un sous-ensemble de traits paraît finalement discriminant, en tout cas pour tracer les contours des classes principales. En premier lieu, la structure actancielle, certains paramètres aspectuels comme le caractère ponctuel/duratif et le contrôle ainsi que deux paramètres secondaires - manifestation et verbalisation. Dans notre typologie, les noms d'affect se distinguent ainsi surtout selon deux axes: (a) le N_affect a un caractère interpersonnel ou non et/ou une cause ; (b) le N_affect est ponctuel et/ou duratif.

Cependant, comme le montrent notre étude et la comparaison avec la classification automatique, certains paramètres sémantiques doivent encore être intégrés pour affiner cette première esquisse de classement : (a) l'intensité, (b) la polarité, dont on a vu l'influence sur la combinatoire de contrôle et les manifestations physiques et (c) la dimension axiologique, essentielle pour les N_affect à caractère social comme mépris ou pitié. Il faudra done vérifier dans quelle mesure la méthode employée peut rendre compte de manière satisfaisante de ces paramètres, peut-être moins directement associés à des propriétés combinatoires. 


\section{Annexe A :}

\section{Collocatifs examinés pour déterminer les dimensions sémantiques}

\begin{tabular}{|c|c|c|c|}
\hline Type & \multicolumn{2}{|c|}{ Dimension } & Collocatifs verbaux et nominaux \\
\hline \multirow[t]{5}{*}{ aspect } & \multicolumn{2}{|l|}{ duratif } & $\begin{array}{l}\text { - moment. jours, journées de N_affect } \\
\text { - } X \text { (agent/expérienceur) être dans. vivre dans, nagor dans Det N affect }\end{array}$ \\
\hline & \multicolumn{2}{|l|}{ ponctuel } & $\begin{array}{l}\text { - bouffée, flux, pointe. flambée, éruption, éclair, élan, accès, transport, explosion } \\
\text { - éclate, explose }\end{array}$ \\
\hline & \multirow[t]{3}{*}{ phasique } & inchoatif & $\begin{array}{l}\text { - Det N_affect naît, s'empare, gagne, envahit, prend, saisit } \\
\text { - X (agent/expérienceur) contracte, noue, se met en, entre dans (Det) N_affect }\end{array}$ \\
\hline & & continuatif & $\begin{array}{l}\text { - Des N_affect dure, persiste, continut } \\
\text { - X entretient, maintient, cultwe Det N_affect }\end{array}$ \\
\hline & & terminatif & $\begin{array}{l}\text { - Det } N \text { _affect se dissipe, s'évanouit, s'apaise, passe } \\
\text { - X (agent/expérienceur) perd Det } N_{-} \text {a affect }\end{array}$ \\
\hline \multirow[t]{2}{*}{ contrôle } & \multicolumn{2}{|c|}{ de l'émotion } & $\begin{array}{l}\text { X (agent/expèrienceur) calme, comprime, contient, domine, dompte, exorcise, } \\
\text { mẩitrise, modère, refoufe, réfrène, rêprime, retient, surmonte, vainc Det N_affect }\end{array}$ \\
\hline & \multicolumn{2}{|c|}{ de la manifestation } & - X (agent/expérienceur) cache, rait, masque, dissinule Dat N_affect \\
\hline \multirow[t]{5}{*}{ manifestation } & \multicolumn{2}{|c|}{ expression du visage } & $\begin{array}{l}\text { - expression, regard de N_affect } \\
\text { - Det N_affect empourpre, enflamme, entaidit X (agent/expérienceur) } \\
\text { - X (agent/expérienceur) blêmit, est hébété, exulte, grimace, irradie, pâlit, } \\
\text { rayonne, resplendit, rougigt. verdit de N_affect }\end{array}$ \\
\hline & \multicolumn{2}{|c|}{ physique "active" } & $\begin{array}{l}\text { - bond, geste, élan de N_affect } \\
\text { - X (agent/expérienceur) bondit, chamte, court, éclate, gambade, recule, saute, } \\
\text { s'écarte, se dresse de N_affect }\end{array}$ \\
\hline & \multicolumn{2}{|c|}{ physique "subie" } & $\begin{array}{l}\text { - frisson, tremblement. larme de } \mathrm{N} \text { affect } \\
-\mathrm{X} \text { (agent/expérienceur) baille, bout, défaille, écume, est comvlsé, frénit, fris- } \\
\text { sonne, grelotce, pleure, sëranouit, sur, suffoque, tremble. tressaillit de } \mathrm{N} \text { affect }\end{array}$ \\
\hline & \multicolumn{2}{|l|}{ effet subi } & $\begin{array}{l}\text { - Det N_affect étrangle, doue, consume, convulse, dérore, étreint, frappe, glace, } \\
\text { habite, hérisse, mine, mord, oppresse, paralyse, pétrifie, plonge, ronge, saisit, } \\
\text { soutève, tenaille, transporte X (agent/expérienceur) [souvent au passif : X est } \\
\text { paralysé de peut] }\end{array}$ \\
\hline & \multicolumn{2}{|l|}{ verbales } & $\begin{array}{l}\text { - chuchorement, cri, rugissement, hurlemient. génissement de N_affect } \\
\text { - X (agent'expérienceur) hurle, crie, rugit de N_affect }\end{array}$ \\
\hline \multirow[t]{3}{*}{ causativité } & \multicolumn{2}{|l|}{ neutres } & - $\bar{X}$ (cause) cause, donле, fait, impose, imspire, procure, provoque Det $N_{-}$affect \\
\hline & \multicolumn{2}{|c|}{ phasiques } & $\begin{array}{l}\text { - X (cause) éveille, déclenche, engendre, entretient, maintiemt, met en, réveille, } \\
\text { brise. chasse, dénoue. dissipe, libèse Det N_affect }\end{array}$ \\
\hline & \multicolumn{2}{|c|}{$\begin{array}{l}\text { "intensifs " } \\
\text { croissance/décroissance }\end{array}$} & $\begin{array}{l}\text { - (cause) accentue, apaise, attise, augmente, excite, favorise, ramime, rawive, } \\
\text { renforce, calme Det N_affect }\end{array}$ \\
\hline \multirow[t]{2}{*}{ verbalisation } & \multicolumn{2}{|c|}{ " expressive " } & - X (agent/expérienceur) hute. crie, épanche Det_poss_N_affect \\
\hline & \multicolumn{2}{|c|}{ " communicative " } & $\begin{array}{l}\text { - X (agent/expérienceur) avoue, exprime, raconte, confie, dit, témoigne, déclare, } \\
\text { communique, dit, partage, expase, fait part de sa Det_poss N_affect }\end{array}$ \\
\hline
\end{tabular}

